\title{
Article \\ Building 2D Model of Compound Eye Vision for Machine Learning
}

\author{
Artem E. Starkov ${ }^{1}$ (D) and Leonid B. Sokolinsky $2, t, \ddagger, *(\mathbb{D})$ \\ 1 South Ural State University (National Research University); starkovae@susu.ru \\ 2 South Ural State University (National Research University); leonid.sokolinsky@susu.ru \\ * Correspondence: leonid.sokolinsky@susu.ru \\ † Current address: 76, Lenin prospekt, Chelyabinsk, Russia, 454080 \\ $\ddagger$ These authors contributed equally to this work.
}

check for updates

Citation: Starkov, A.E.; Sokolinsky, L.B. Building 2D Model of Compound Eye Vision for Machine Learning. Preprints 2021, 1, 0. https://doi.org/

\section{Received:}

Accepted:

Published:

Publisher's Note: MDPI stays neutral with regard to jurisdictional claims in published maps and institutional affiliations.

\begin{abstract}
This paper presents a two-dimensional mathematical model of compound eye vision. Such a model is useful for solving navigation issues for autonomous mobile robots on the ground plane. The model is inspired by the insect compound eye that consists of ommatidia, which are tiny independent photoreception units, each of which combines a cornea, lens, and rhabdom. The model describes the planar binocular compound eye vision, focusing on measuring distance and azimuth to a circular feature with an arbitrary size. The model provides a necessary and sufficient condition for the visibility of a circular feature by each ommatidium. On this basis, an algorithm is built for generating a training data set to create two deep neural networks (DNN): the first detects the distance, and the second detects the azimuth to a circular feature. The hyperparameter tuning and the configurations of both networks are described. Experimental results showed that the proposed method could effectively and accurately detect the distance and azimuth to objects.
\end{abstract}

Keywords: robot vision; compound eye; two-dimensional model; distance measurement; azimuth measurement; deep learning; training data set generation; deep neural network

\section{Introduction}

Robotics is a vibrant field that grows in importance from year to year. Robots can be classified according to the environment in which they operate and the mechanism of interaction. The most common distinction is between fixed and mobile robots. These two types of robots have very different working environments and therefore require very different capabilities. Fixed robots are mostly industrial robotic manipulators that work in well-defined environments adapted for robots. By contrast, mobile robots are expected to move around and perform tasks in large, ill-defined, and uncertain environments that are not explicitly designed for robots. They need to deal with situations that are not precisely known in advance and that change over time [1]. Mobile robots are divided into two classes: non-autonomous and autonomous [2]. The non-autonomous mobile robots rely on operator control. They use their sensors to give their operator remote access to dangerous, distant, or inaccessible places. Fully autonomous mobile robots do not rely on an operator. But instead, they make decisions on their own and perform tasks while navigating uncertain terrain and in a constantly changing environment.

A crucial element of mobile robots is the use of sensors. One of the most important sensors in robotics is the distance sensor that measures the distance from the robot to an object. By using multiple distance sensors or by rotating the sensor, the angle of the object relative to the front of the robot can be measured. The distance sensor is classified as active or passive [3]. An active sensor affects the environment by emitting energy: it transmits a signal and then receives its reflection from an object. One way of determining distance is to measure the time that elapses between sending a signal and receiving it (ultrasound and laser sensors) [4]. The infrared sensors use another way of estimating the distance: light intensity decreases with the square of the distance from the source, and this relationship is used to measure the approximate distance to the object. The third way of reconstructing the distance is by using triangulation (laser triangulation sensors) [5]. 

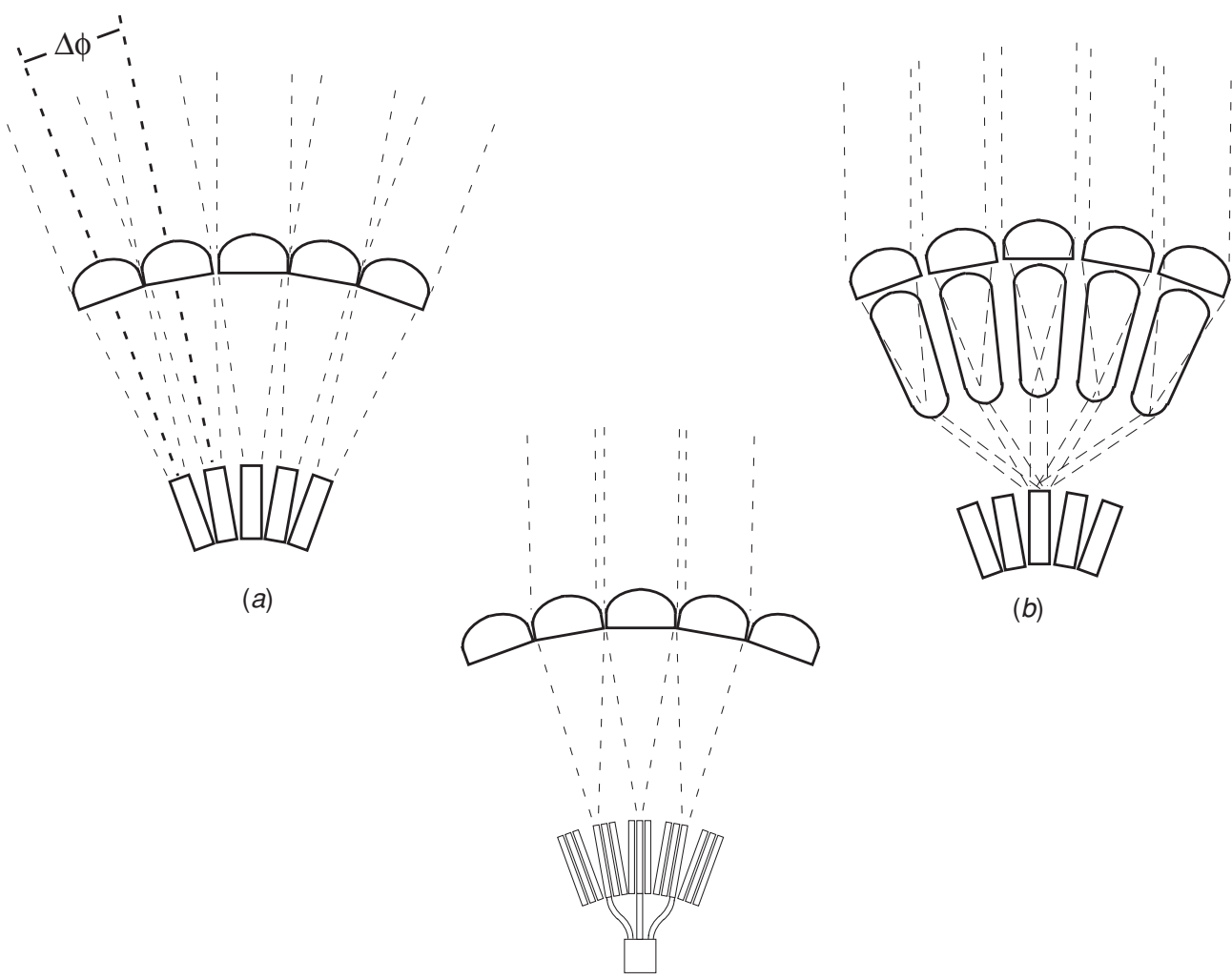

(b)

$(c)$

Figure 1. Insect vision configurations: (a) apposition, (b) superposition, and (c) neural superposition [8].

Active sensors have two common disadvantages: first, they consume additional energy to generate rays, and second, a robot with active sensors can be detected by an external observer, which is not always permissible. A passive sensor does not affect the environment: it simply perceives the light reflected off an object. A well-known example of passive sensors is a digital camera [5]. The distance between the object and an observer can be calculated by using visual information gained from a pair of images taken by two cameras, which is known as stereo image [6]. A stereo camera requires sophisticated controls, including panning, tilting, zooming, focusing on, and tracking a moving object [7]. For this reason, the use of stereo cameras in fully autonomous robots is difficult.

Video sensors inspired by compound eyes of insects are promising alternatives to digital cameras [9]. Such video sensors have no moving parts and do not require any control. The insect vision has the following three basic configurations: apposition, superposition, and neural superposition compound eyes [8]. Each configuration has its advantages and disadvantages. Apposition compound eyes are composed of hundreds up to tens of thousands of microlens - receptor units referred to as ommatidia arranged on a curved surface (Figure 1a). Each ommatidium consists of a microlens (facet lens) and a small group of rhabdomere (photoreceptor) bundles, called the rhabdom. Pigments form opaque walls between adjacent ommatidia to avoid light which is focused by one microlens being received by an adjacent channel's receptor [9]. There are no moving or dynamically transforming parts in the apposition compound eye, and it does not need to be controlled by the nervous system. The spatial acuity of the apposition compound eye is determined by the interommatidial angle $\Delta \phi=D / R$, where $D$ is the diameter of the facet lens and $R$ is the local radius of curvature of the eye [10]. The superposition eye gathers light beams from adjacent ommatidia (Figure 1b). This effectively enhances the photosensitivity but reduces the effective acuity due to the blurring effect. In the neural superposition eye, one rhabdomere in several adjacent ommatidia shares an overlapped field of view with the other (Figure 1c). Large amounts of summation can provide the opportunity to increase 
signal-to-noise ratios. Therefore, overlapped fields provide a motion resolution greater than that implied by the facet spacing of the compound eye, a phenomenon known as hyperacuity [11].

The apposition vision configuration is the most promising for robotics because of its simplicity. In recent years, great progress has been achieved in the design of video sensors inspired by the artificial composite eyes of this structure [12]. The first artificial compound eye, constructed in 1991, had a weight of $1 \mathrm{~kg}$, a diameter of $23 \mathrm{~cm}$, and consisted of 118 artificial ommatidia with a facet diameter of $6 \mathrm{~mm}[13,14]$. The artificial compound eye, created in 2013, has a weight of 1.75 grams, a diameter of $12.8 \mathrm{~mm}$, and consists of 630 artificial ommatidia with a facet diameter of $172 \mu \mathrm{m}$ [15]. Modern technologies allow creating the curved microlens arrays with a diameter of $500 \mu \mathrm{m}$, consisting of microlens with a diameter of $20 \mu \mathrm{m}$ [16].

Artificial neural network (ANN) $[17,18]$ is one of the most promising and rapidly developing techniques used to control the autonomous mobile robot behavior. This technology is used for navigation [19-21], object detection and recognition [22,23], obstacle avoidance $[24,25]$, autonomous driving [26,27] and other applications. One of the main factors limiting the use of ANNs in robotics is the need to create large annotated data sets (up to one hundred thousand copies) for training a neural network [28]. In many use cases collecting or labeling data is very difficult or not possible at all [29]. One more factor limiting the design of robotic imaging systems based on compound eyes is the necessity to use expensive facilities and complicated fabrication procedures for manufacturing compound vision sensors [30]. Mathematical modeling and computer simulation of compound eye vision systems are effective ways to overcome these limitations.

In this paper, we present a mathematical model of the planar binocular compound eye vision, focusing on measuring distance and azimuth to a circular feature with an arbitrary size. The model provides a necessary and sufficient condition for the visibility of a circular feature by each ommatidium. On this basis, an algorithm is built for generating a training data set. We used the generated data set to create two ANNs: the first detects the distance, and the second detects the azimuth to a circular feature. The rest of the paper is organized as follows. Section 2 provides a review of known methods for measuring the distance and azimuth to an object using passive optical sensors. In Section 3, we present a mathematical model of the planar compound eye vision. Section 4 is devoted to the issues of generating a training set based on the presented model of planar compound eye vision. Section 5 describes two deep neural networks that calculate the distance and azimuth to a circular feature based on data yielded by a planar binocular compound eye system. In Section 6, we describe the experiments and have a discussion based on the results. Section 7 summarizes the obtained results and outlines the directions of future research.

\section{Related Work}

This section presents an overview of works devoted to mathematical models and methods for measuring distance and azimuth to an object based on passive optical sensors. One of the simplest methods is bearing-based distance measurement [31]. This method (see Figure 2) calculates distance $d_{\text {object }}$ to an object from known height $h_{\text {camera }}$ of the observing camera, angle $\varphi$ of the camera tilt, and height $h_{\text {object }}$ of the object:

$$
d_{\text {object }}=\tan (\varphi) \cdot\left(h_{\text {camera }}-h_{\text {object }}\right) .
$$

This simple model is only valid when $h_{\text {camera }}>h_{\text {object }}$ and $\varphi<\frac{\pi}{2}$. In addition, we need to know height $h_{\text {object }}$ of the object, which is not always feasible in practice. The measurement error as a function of $\Delta \varphi$ can be estimated as follows:

$$
d_{\text {error }}(\Delta \varphi)=\left|(\tan (\varphi+\Delta \varphi)-\tan (\varphi)) \cdot\left(h_{\text {camera }}-h_{\text {object }}\right)\right|
$$




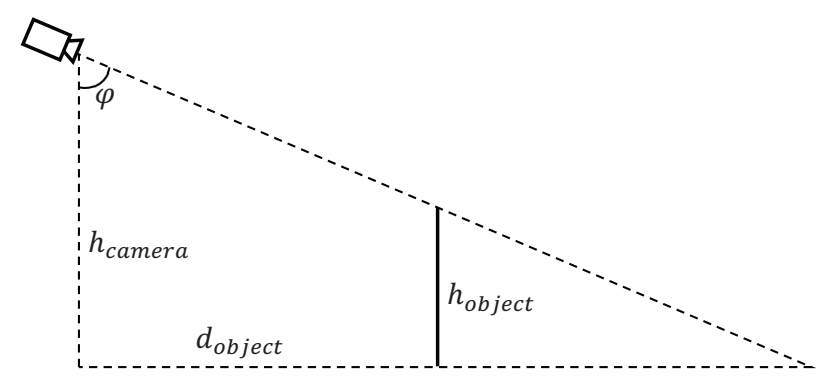

Figure 2. Simple bearing-based distance measurement model

It is obvious from this equation that the error is rising exponentially for positive $\Delta \varphi$ with fixed $h_{\text {camera }}$ and $h_{\text {object }}$. Thus, this method is not applicable when the height of the camera is comparable to the height of the object.

In [32-34], a monocular vision model is proposed for determining the 3D position of circular and spherical features. This model uses $2 \mathrm{D}$ image representing a perspective projection of a feature and the effective focal length of the camera to find the feature's location with respect to the camera frame (see Figure 3). The described method can be generalized for 3D quadratic features such as ellipsoid, paraboloid, hyperboloid, and cylindroid, but not for features of arbitrary shape. In addition, this method has a relatively high computational complexity and cannot provide sufficient accuracy in measuring the distance to the visible object.

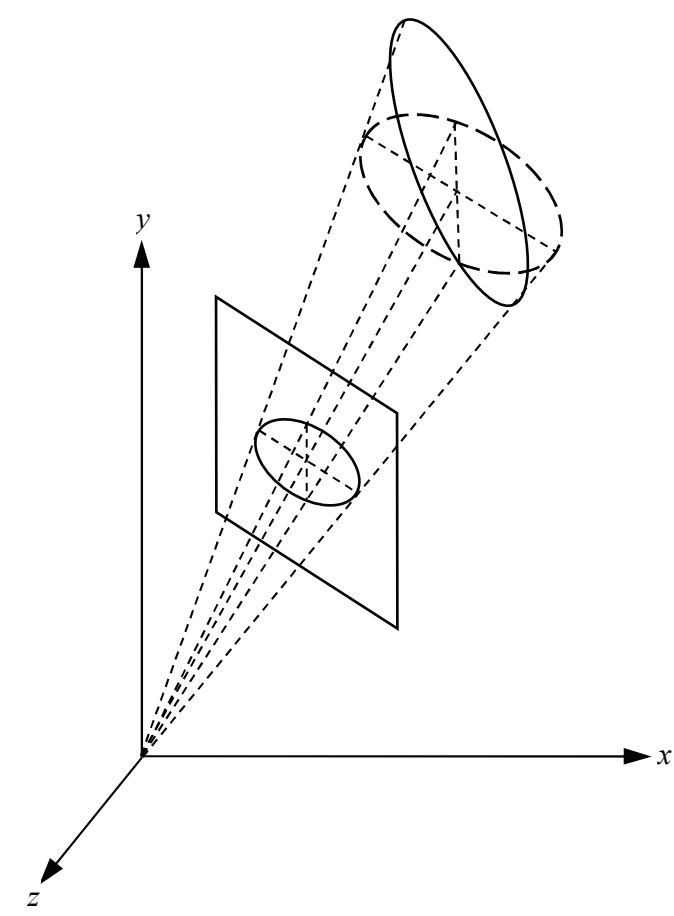

Figure 3. Perspective projection of circular feature (adapted from [34])

The next method for determining the distance to a visible object is based on using two video cameras having the same specification, which are conjugated in a certain way to generate a stereo image in the form of two 2D images [35-39]. This method is based on epipolar geometry $[40,41]$. The simplest model of the binocular stereo vision system is shown in Figure 4. In this model, the image sensor matrices are located in the same plane perpendicular to the epipolar plane (the epipolar plane is the plane defined by points $P, P_{l}$, and $P_{r}$ in Figure 4a). In this case, distance $d$ between point $P$ and the center of the stereo camera system can be calculated as follows (see Figure $4 \mathrm{~b}$ ). Since triangles $\Delta P P_{l} P_{r}$ 


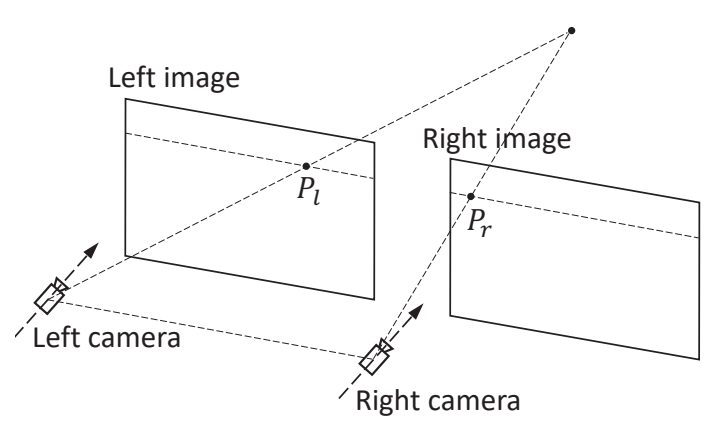

(a)

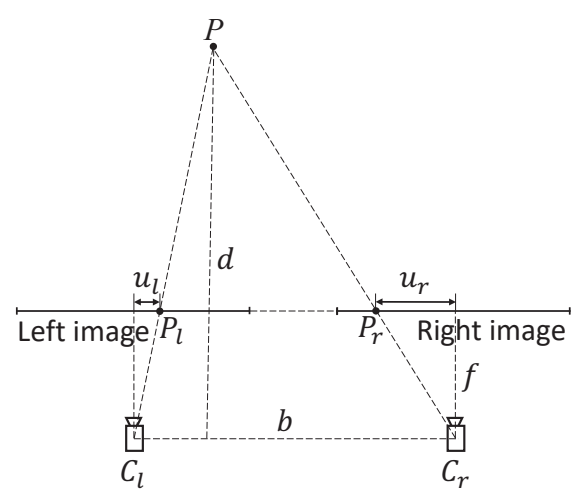

(b)

Figure 4. (a) Simple binocular model; (b) 2D projection of binocular model (adapted from [35])

and $\triangle P C_{l} C_{r}$ are similar, the ratio of the corresponding edges is equal to the ratio of the corresponding heights:

$$
\frac{b-\left(u_{l}+u_{r}\right)}{b}=\frac{d-f}{d}
$$

where $f$ is the focal length. This can be transformed into

$$
d=\frac{b f}{u_{l}+u_{r}} .
$$

To find the object of interest in the left image, we must perform the object segmentation procedure [41]. This gives us point $P_{l}$. To find the corresponding point $P_{r}$ on the right image, we must perform the stereo matching procedure [42] for both images. And first of all, we must perform a careful camera calibration process [43]. All this makes it difficult to use this method for autonomous mobile robots. In addition, this technique also cannot provide high measurement accuracy.

Another passive method to measure the distance to an object is to use a plenoptic camera [45]. A plenoptic or light-field camera acts like a microcamera array that records not only the light intensity but a combination of light intensity and the direction of incident light rays. The distance estimation is based on disparities observed in neighboring microlens images, similar to stereo camera approaches. The plenoptic camera can give 3D information for every point of the scene with one camera, one main lens, and a microlens array placed directly in front of the image sensor. The price that has to be paid for these additional features is a significant reduction in the effective image resolution [46]. A geometric optical model to measure the distance to an object using a light field image is proposed in [44].

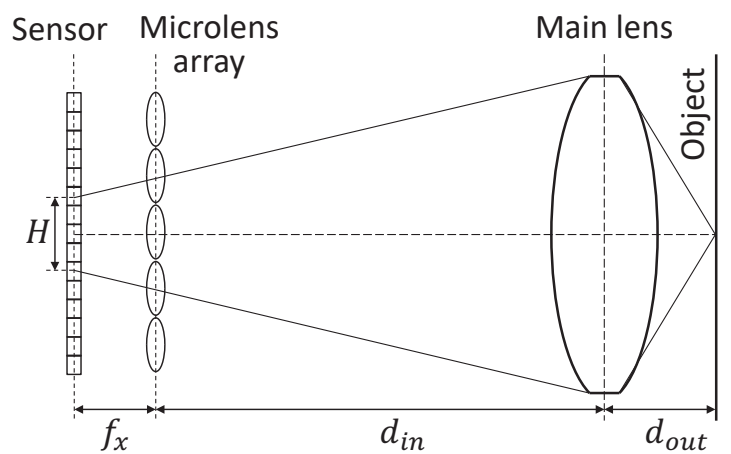

(a)

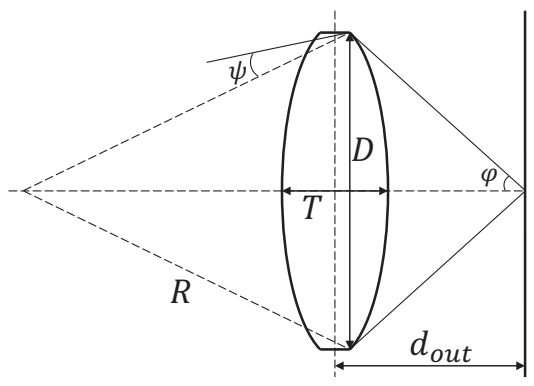

(b)

Figure 5. (a) Plenoptic camera model; (b) Light ray propagation (adapted from [44]) 


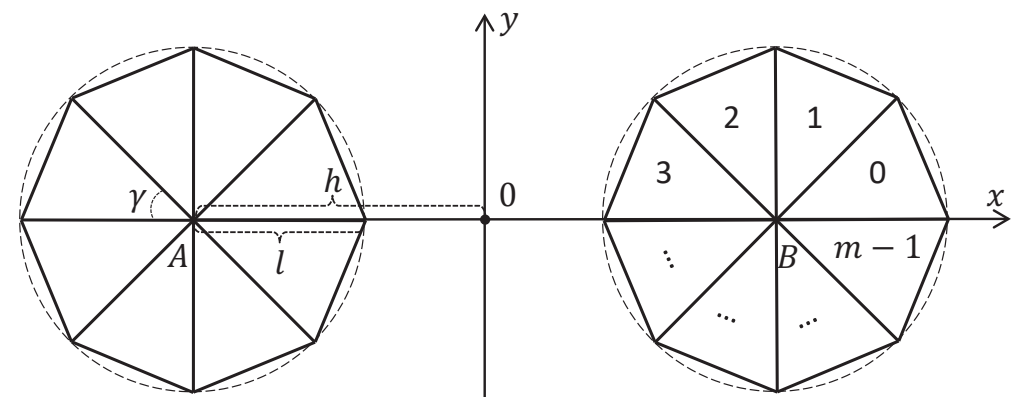

Figure 6. The 2D model of binocular compound eye vision

This model is shown in Figure 5 a. The distance $d_{\text {out }}$ between main lens and an object can be calculated by the following equation:

$$
d_{\text {out }}=\frac{D}{2 \tan \varphi}+\sqrt{R^{2}+D^{2} / 4}-R+T / 2,
$$

where $R$ represents the radius of curvature of main lens; $T$ represents the central thickness of main lens and $D$ is the pupil diameter of main lens (see Figure $5 \mathbf{b}$ ). The angle $\varphi$ is calculated by

$$
\varphi=\arcsin \left(n_{1} \sin \psi\right)-\arcsin \left(\frac{D}{2 R}\right),
$$

where $n_{1}$ is the refractive index of the main lens; $\psi$ is the included angle between the normal, the dashed line, and the refractive light rays in the main lens. The refractive angle $\psi$ can be calculated by the following known camera parameters: the focal length $f_{x}$ of microlens array, the distance $d_{\text {in }}$ between the microlens array and main lens, and the length $H$ of corresponding light field [44]. The described method also requires a complex camera calibration process [46], does not provide a high measurement accuracy at long distances [47], and it is poorly suited for autonomous mobile robots.

The review of related papers shows a lack of works devoted to mathematical models of distance measurement using artificial binocular compound eye vision systems. At the same time, the progress made in manufacturing artificial compound eyes and their unique features makes this issue urgent.

\section{Two-Dimensional Model of Compound Eye Vision}

The 2D model of binocular compound eye vision is presented in Figure 6. The model includes two circular compound eyes located symmetrically relative to the $y$-axis, the centers $A$ and $B$ of which lie on the $x$-axis. The distance from the origin to the center of each eye is equal to $h$. The composite eye consists of $m$ ommatidia, represented as equal-sized isosceles triangles. The legs have the length $l$, and the base has the length $s$. The angle between the legs is equal to $\gamma$. It is obvious that

$$
\gamma=\frac{2 \pi}{m} \text {. }
$$

In this model, we assume that $h>l$, and $m \geqslant 4$. Taking into account 1 , it follows

$$
\gamma \leqslant \frac{\pi}{2}
$$

Let us number the ommatidia counterclockwise from 0 to $m-1$, starting with the ommatidium, which lies on the $x$-axis (see the right eye in Figure 6). In the model, the ommatidium field of view is defined as a solid angle bounded by its legs. In Figure 7a, the gray solid angle depicts the field of view of the ommatidium with number 1 . If a point lies on the bound between adjacent ommatidia, then it is visible only for the ommatidium with the larger 


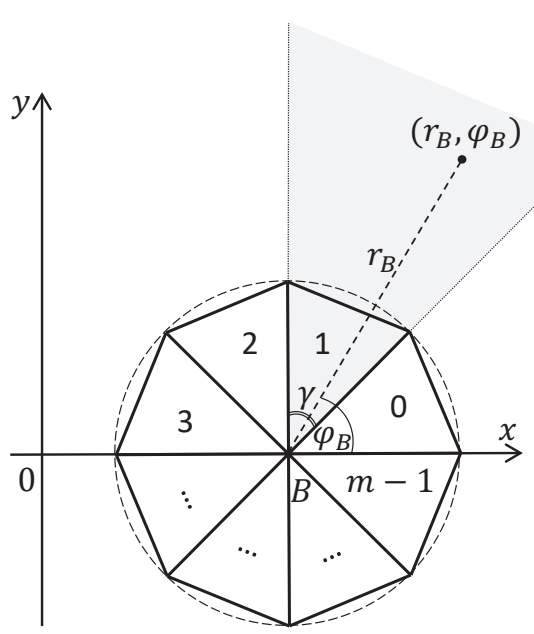

(a)

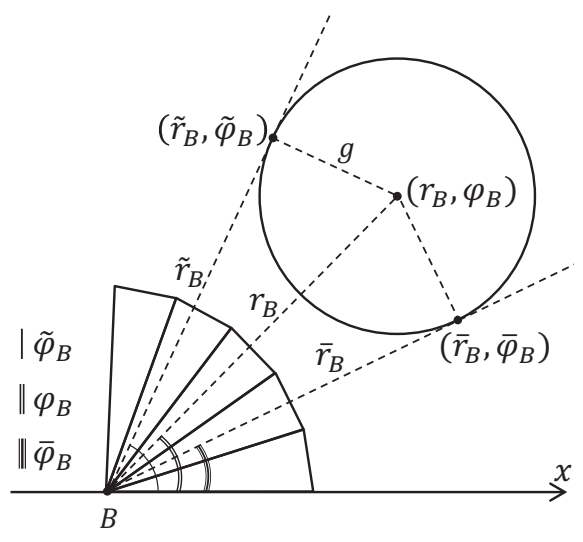

(b)

Figure 7. Ray tracing: (a) point; (b) circular feature

number. Thus, the fields of view of different ommatidia do not intersect, and their union forms a solid angle of $360^{\circ}$.

Let us build a ray tracing model. Consider the polar coordinate system $\left(r_{B}, \varphi_{B}\right)$ with the origin in the point $B$ and the angle measured from the $x$-axis. The following proposition gives an equation to calculate the number of ommatidia, which observes the point with given polar coordinates.

Proposition 1. Let the point $\left(r_{B}, \varphi_{B}\right)$ be given in polar coordinates with center $B$ (see Figure $7 a$ ). The number $k$ of the ommatidium, to whose field of view the point $\left(r_{B}, \varphi_{B}\right)$ belongs, is determined by the equation

$$
k=\left\lfloor\frac{m}{2 \pi} \varphi_{B}\right\rfloor
$$

Proof. It follows from equation (1) that the angle $\varphi_{B}$ must satisfy the system of inequalities

$$
\left\{\begin{array}{l}
\varphi_{B} \geqslant \frac{2 \pi}{m} k \\
\varphi_{B}<\frac{2 \pi}{m}(k+1) .
\end{array}\right.
$$

Convert this system to the form

$$
\left\{\begin{array}{l}
k \leqslant \frac{m}{2 \pi} \varphi_{B} \\
k>\frac{m}{2 \pi} \varphi_{B}-1
\end{array}\right.
$$

By the definition of the greatest integer less than or equal to $\frac{m}{2 \pi} \varphi_{B}$, it follows

$$
k=\left\lfloor\frac{m}{2 \pi} \varphi_{B}\right\rfloor .
$$

The next Proposition extends Proposition 1 for the case of circular features.

Proposition 2. Let a circular feature with radius $g$ and center $\left(r_{B}, \varphi_{B}\right)$ in polar coordinates with center $B$ be given (see Figure $7 b$ ). This circular feature or part of it belongs to the field of view of the ommatidium with the number $k$ if and only if

$$
\left\lfloor\frac{m}{2 \pi}\left(\varphi_{B}-\arcsin \left(\frac{g}{r_{B}}\right)\right)\right\rfloor \leqslant k \leqslant\left\lfloor\frac{m}{2 \pi}\left(\varphi_{B}+\arcsin \left(\frac{g}{r_{B}}\right)\right)\right\rfloor .
$$


Proof. Consider the tangents to the circle at the points $\left(\bar{r}_{B}, \bar{\varphi}_{B}\right)$ and $\left(\tilde{r}_{B}, \tilde{\varphi}_{B}\right)$ passing through the point $B$ in Figure $7 \mathrm{~b}$. We have

$$
\bar{\varphi}_{B}=\varphi_{B}-\arcsin \frac{g}{r_{B}}
$$

and

$$
\tilde{\varphi}_{B}=\varphi_{B}+\arcsin \frac{g}{r_{B}} .
$$

Taking into account Proposition 1, it follows that

$$
\left\lfloor\frac{m}{2 \pi}\left(\varphi_{B}-\arcsin \left(\frac{g}{r_{B}}\right)\right)\right\rfloor \leqslant k \leqslant\left\lfloor\frac{m}{2 \pi}\left(\varphi_{B}+\arcsin \left(\frac{g}{r_{B}}\right)\right)\right\rfloor .
$$

Definition 1. In the context of the model under consideration, an object is invisible to the compound eye if and only if it lies in the field of view of only one ommatidium.

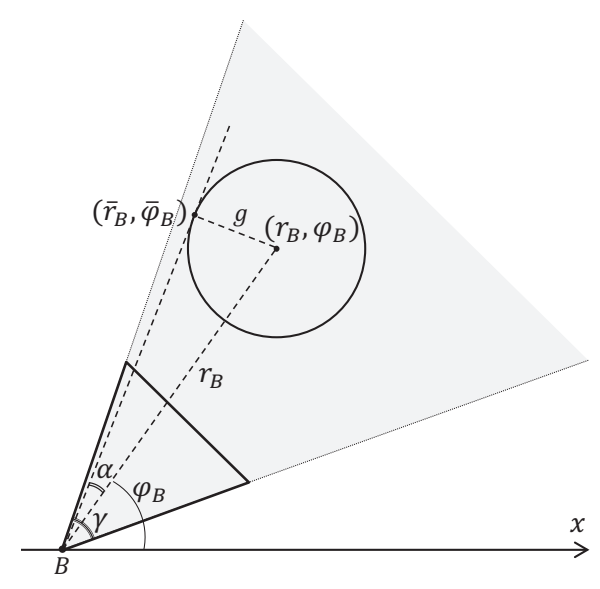

Figure 8. Invisible circular feature

The following proposition gives us a sufficient condition for the visibility of the circular feature.

Proposition 3. Let a circular feature with radius $g$ and center $\left(r_{B}, \varphi_{B}\right)$ in polar coordinates with center $B$ be given. Let $\gamma$ be the view field angle of an ommatidium. The inequality

$$
g>r_{B} \sin \frac{\gamma}{2}
$$

is a sufficient condition for the visibility of the circular feature.

Proof. Let us assume the opposite: the circular feature with radius $g$ and center $\left(r_{B}, \varphi_{B}\right)$ is invisible, and inequality (4) holds. Consider the tangent to the circle at the point $\left(\bar{r}_{B}, \bar{\varphi}_{B}\right)$ passing through the point $B$ in Figure 8 . Let $\alpha$ be the angle between this tangent and the ray from the point $B$ to the point $\left(r_{B}, \varphi_{B}\right)$. Taking into account Definition 1 , inequality (2) and inequality (4), we have

$$
g=r_{B} \sin \alpha \leqslant r_{B} \sin \frac{\gamma}{2}<g
$$

Thus we have a contradiction.

Definition 2. In the context of the model under consideration, the binocular field of view (BFV) is the solid angle $\theta$ between the tangents to the compound eye circles drawn from the origin in the direction of the positive y-axis (see Figure 9).

$\mathrm{BFV}$ is uniquely determined by the angle $\psi$ between the right tangent and the $\mathrm{x}$ axis. Obviously,

$$
\psi=\arcsin \frac{l}{h} .
$$

It follows

$$
\theta=\pi-2 \arcsin \frac{l}{h}
$$




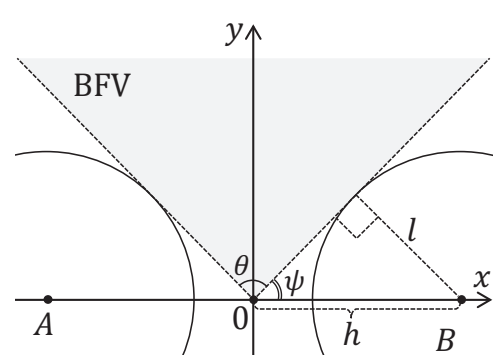

Figure 9. Binocular field of view (BFV)

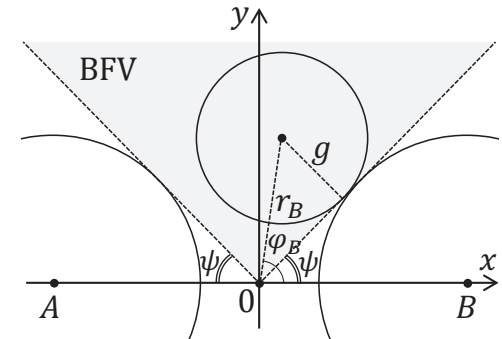

Figure 10. A circle feature lying in BFV

BFV has the following three important properties.

Property 1. Any object lying in BFV does not cross the compound eyes.

Property 2. Any object lying in the BFV is entirely visible to both compound eyes.

Property 3. Any circular feature lying in BFV is invisible to all ommatidia located in the negative region of the $y$-axis.

The following proposition gives us a necessary and sufficient condition for a circular feature to lie entirely in BFV.

Proposition 4. Let a circular feature with the radius $g$ and the center $(r, \varphi)$ in the polar coordinates centered at the origin be given $(0 \leqslant \varphi<2 \pi)$. This circular feature lies entirely inside the BFV defined by the angle $\psi$ if and only if

$$
\psi+\arcsin \frac{g}{r} \leqslant \varphi \leqslant \pi-\psi-\arcsin \frac{g}{r} .
$$

Proof. Consider a circular feature with the radius $g$ and the center $(r, \varphi)$ that touches the right bound of BFV in the Figure 10. Using right triangle properties, we have

$$
g=r \sin (\varphi-\psi)
$$

It follows

$$
\varphi=\psi+\arcsin \frac{g}{r} .
$$

Hence, a circular feature with the radius $g$ and the center $(r, \varphi)$ lies to the left of the right BFV bound if and only if

$$
\varphi \geqslant \psi+\arcsin \frac{g}{r} .
$$

In the same way, we obtain that a circular feature with the radius $g$ and the center $(r, \varphi)$ lies to the right of the left BFV bound if and only if

$$
\varphi \leqslant \pi-\psi-\arcsin \frac{g}{r} .
$$

Below, in Algorithm 1, we will need equations that convert the polar coordinates $(r, \varphi)$ centered at the origin to the polar coordinates $\left(r_{A}, \varphi_{A}\right)$ centered at the point $A=(0,-h)$ for the left compound eye, and to the polar coordinates $\left(r_{B}, \varphi_{B}\right)$ centered at the point $B=(0, h)$ for the right compound eye. The following proposition provides us with such equations. 


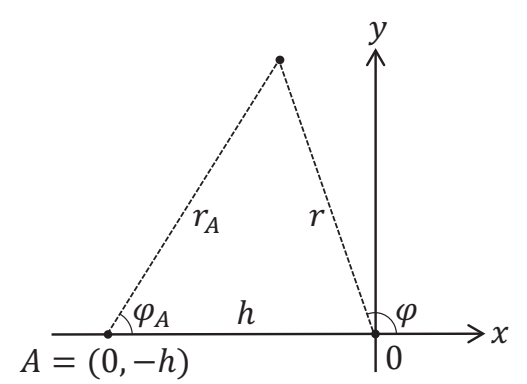

(a)

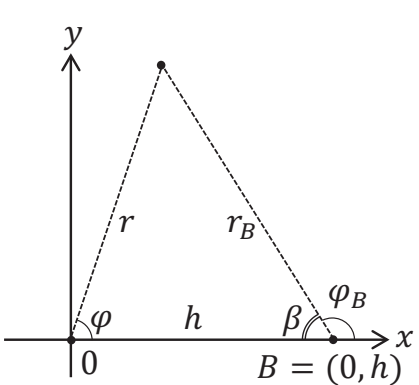

(b)

Figure 11. Converting polar coordinates: (a) left eye, (b) right eye

Proposition 5. Let $0 \leqslant \varphi \leqslant \pi, 0<h, 0<r$ be given. For the polar coordinates $(r, \varphi)$ centered at the origin, the following equations convert them to the polar coordinates $\left(r_{B}, \varphi_{B}\right)$ centered at the point $B=(0, h)$, and to the polar coordinates $\left(r_{A}, \varphi_{A}\right)$ centered at the point $A=(0,-h)$ :

$$
\begin{gathered}
r_{B}=\sqrt{h^{2}+r^{2}-2 h r \cos \varphi} ; \\
\varphi_{B}=\pi-\arccos \frac{h-r \cos \varphi}{\sqrt{h^{2}+r^{2}-2 h r \cos \varphi}} ; \\
r_{A}=\sqrt{h^{2}+r^{2}+2 h r \cos \varphi} ; \\
\varphi_{A}=\arccos \frac{h+r \cos \varphi}{\sqrt{h^{2}+r^{2}+2 h r \cos \varphi}} .
\end{gathered}
$$

Proof. In the context of Figure 11b, by the law of cosines, we have:

$$
\begin{gathered}
r_{B}=\sqrt{h^{2}+r^{2}-2 h r \cos \varphi} ; \\
\beta=\arccos \frac{h^{2}+r_{B}^{2}-r^{2}}{2 h r_{B}} .
\end{gathered}
$$

Taking into account that $\beta=\pi-\varphi_{B}$, it follows

$$
\varphi_{B}=\pi-\arccos \frac{h-r \cos \varphi}{\sqrt{h^{2}+r^{2}-2 h r \cos \varphi}} .
$$

In the same way, in the context of Figure 11a, we can obtain equations 10 and 11.

\section{Algorithm for Generating Training Data Set}

Based on the proposed 2D model of binocular compound eye vision, we have developed Algorithm 1 for generating annotated data sets for training artificial neural networks capable of determining the distance and azimuth to the observed objects. The data set $\mathcal{M} \subset \mathbb{R}^{2} \times\{0,1\}^{m / 2} \times\{0,1\}^{m / 2}$ consists of elements of the form $\left(r, \varphi, \Omega_{A}, \Omega_{B}\right)$. Each element corresponds to one observed circular feature with the following four parameters. The pair $(r, \varphi)$ determines the polar coordinates of the circle feature center. The parameter $\Omega_{A}$ is the bit map with the length of $m / 2$ produced by the left compound eye: $\Omega_{A}[i]=1$ if and only if the $i$ th ommatidium of left eye observes the circular feature $(i=0, \ldots, m / 2-1)$. The parameter $\Omega_{B}$ is the bit map with the same length of $m / 2$ produced by the right compound eye: $\Omega_{B}[j]=1$ if and only if the $j$ th ommatidium of right eye observes the circular feature $(j=0, \ldots, m / 2-1)$.

Let us make brief comments on the steps of Algorithm 1. Step 1 performs input of the algorithm parameters:

$h \quad$ : the distance from the origin to the centers of compound eyes (see Figure 6); 


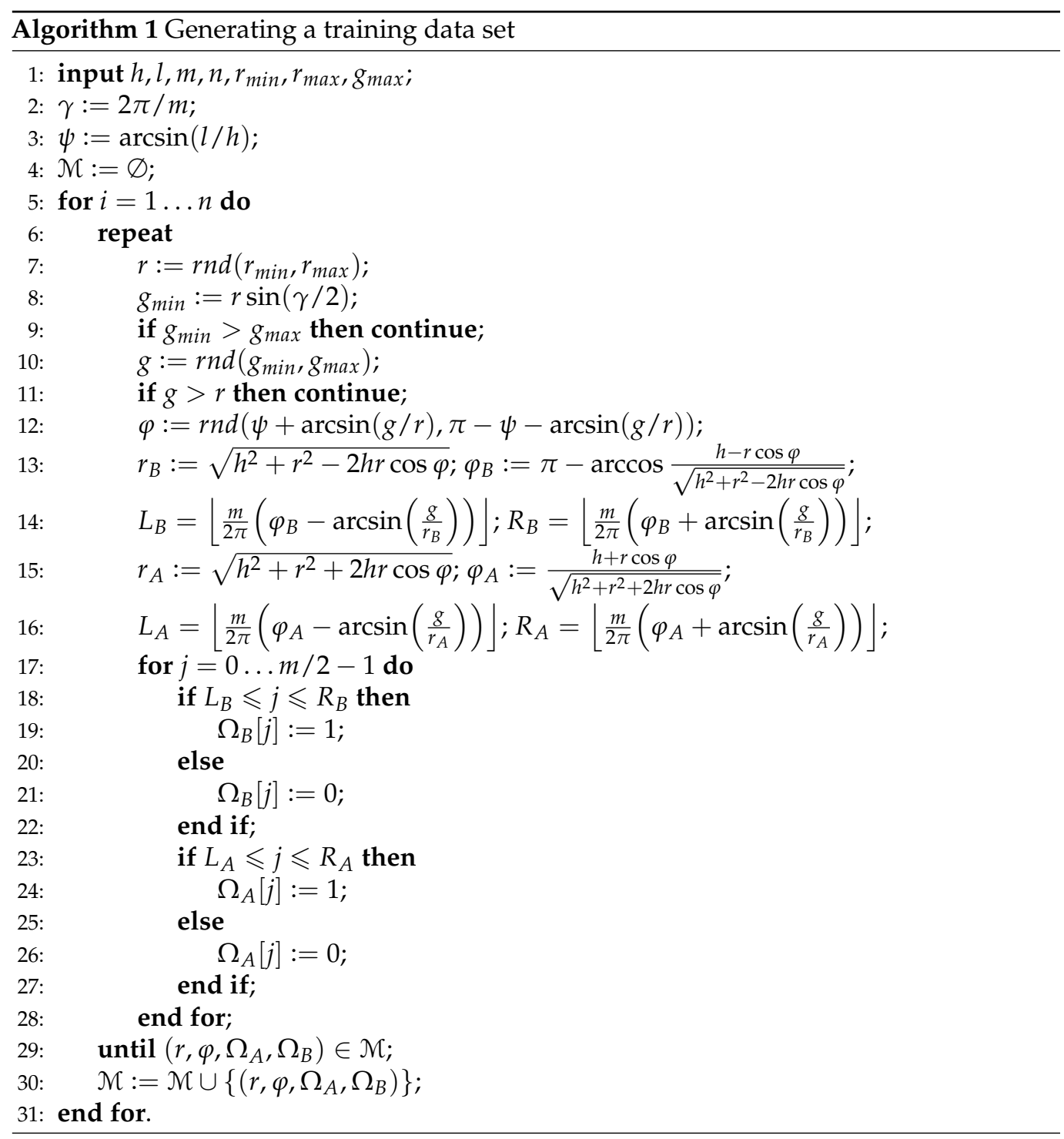

$l \quad:$ the radius of compound eye;

$m$ : the number of ommatidia in compound eye;

$n$ : the number of elements in the training data set;

$r_{\min }:$ the minimum distance from the origin to the center of circular feature;

$r_{\max }:$ the maximum distance from the origin to the center of circular feature;

$g_{\max }:$ the maximum radius of circular feature.

Step 2 calculates the angle $\gamma$ of the ommatidium field of view according to equation (1). In Step 3, the angle $\psi$ of the binocular field of view is calculated using equation (5). In Step 4 , the set $\mathcal{M}$ is initially defined as an empty set. Steps 5-31 implement a for loop that inserts $n$ elements into $\mathcal{M}$. The repeat/until loop (Steps 6-29) generates one new element of the training data set. Step 7 generates the distance $r$ from the origin to the center of circular feature using the $r$ nd function, which calculates a random real number from the interval $\left[r_{\min }, r_{\max }\right]$. Step 8 calculates the minimum radius of circular feature by inequality (4), which provides a sufficient condition for its visibility. Step 9 checks the condition $g_{\min }>g_{\max }$. If this condition is true, then it forces the repeat/until loop to begin the next iteration. Step 10 calculates the radius $g$ of circular feature as a random real number from the interval $\left[g_{\min }, g_{\max }\right]$. Step 11 checks the condition $g>r$. If this condition is true, then it forces the repeat/until loop to begin the next iteration. Step 12 
Table 1: Parameters of model

\begin{tabular}{l|l|l}
\hline $\begin{array}{l}\text { Para- } \\
\text { meter }\end{array}$ & Semantics & Value \\
\hline$m$ & $\begin{array}{l}\text { Number of ommatidia in } \\
\text { compound eye }\end{array}$ & 720 \\
$l$ & $\begin{array}{l}\text { Compound eye radius } \\
\text { Distance between com- } \\
\text { pound eye centers }\end{array}$ & 20 \\
$h$ & $\begin{array}{l}\text { Ommatidium angle of } \\
\text { view }\end{array}$ & $0.05^{\circ}$ \\
$\theta$ & BFV angle & $120^{\circ}$ \\
\hline
\end{tabular}

Table 2: Parameters of objects

\begin{tabular}{|c|c|c|}
\hline $\begin{array}{l}\text { Para- } \\
\text { meter }\end{array}$ & Semantics & Value \\
\hline$r_{\min }$ & $\begin{array}{l}\text { Minimum distance } \\
\text { to circular feature }\end{array}$ & 1000 \\
\hline$r_{\max }$ & $\begin{array}{l}\text { Maximum distance } \\
\text { to circular feature }\end{array}$ & 5000 \\
\hline$g_{\min }$ & $\begin{array}{l}\text { Minimum radius of } \\
\text { circular feature }\end{array}$ & 0.5 \\
\hline$g_{\max }$ & $\begin{array}{l}\text { Maximum radius } \\
\text { of circular feature }\end{array}$ & 500 \\
\hline
\end{tabular}

randomly generates the angle $\varphi$ so that the circular feature of the radius $g$ and the center $(r, \varphi)$ in polar coordinates lies entirely inside the binocular field of view (see Proposition 4 ). Step 13 converts polar coordinates $(r, \varphi)$ to polar coordinates $\left(r_{B}, \varphi_{B}\right)$ using equations 8 and 9. Based on Proposition 2, Step 14 determines, for the right compound eye, the interval $\left[L_{B}, R_{B}\right]$, which includes the numbers of the ommatidia that observe the circular feature. In the same way, Steps 15,16 determine the interval $\left[L_{A}, R_{A}\right]$, which includes the numbers of the ommatidia that observe the circular feature in the case of the left compound eye. Using the obtained data, Steps 17-28 generate the new element $\left(\Omega_{A}, \Omega_{B}, r, \varphi\right)$ to include in the training data set. If the obtained element does not have a duplicate in $\mathcal{M}$, it is added to the training data set in Step 30.

We implemented the described algorithm in the form of web application named CoViDSGen (compound vision data set generator). The web application CoViDSGen is accessible at https:/ /sp.susu.ru/covidsgen. This system is implemented using Python programming language and the Flask web framework [48]. As an implementation of the rnd function invocated in the steps 7, 10, and 12 of Algorithm 1, we used the random.uniform function from the numpy library. The CoViDSGen source code is freely available at https: / / github.com/artem-starkov/covidsgen. CoViDSGen allows you to set the parameters of Algorithm 1 in a dialog box. As a result, you can load a text file in CSV format that includes elements of the training data set.

\section{Design of Deep Neural Networks}

Using the 2D model of binocular compound eye vision presented in Section 3, we investigated the capability of DNN to determine the distance and azimuth to a visible object. To train DNN, we generated a data set in CSV format using Algorithm 1. This data set consists of 100000 elements and is obtained with the parameters presented in Tables 1 and 2. The parameters of the model of compound eye vision system (see Table 1) are comparable to the proportions of the robber fly vision system [49]. The angle $\gamma$ of ommatidium field of view is calculated by equation (1). The angle $\theta$ of BFV is calculated by equation (6). The parameters of observed objects are presented in Table 2. All observed objects are the circular features of different radii located at different distances from the observer. The estimation of $g_{\min }$ is obtained using the equation $g_{\min }=r_{\min } \sin (\gamma / 2)$ inspired by inequality (4). All 100000 elements of the training data set were generated in one pass (single execution) of Algorithm 1. We inspected the obtained data set using a machine learning platform "Weights \& Biases Sweeps (W\&B)" [50]. The result is presented in Figure 12. This histogram shows that most of objects accumulate in the interval $[500,3000]$ of distances to the observer. Such a skew can severely affect the quality of neural network training. The explanation of this anomaly is presented in Figure 13, which shows a diagram of the spatial distribution of object centers in the BFV zone after generation in one pass. For each orbit with radius $r$, Algorithm 1 generates approximately the same number of objects. However, the length of the orbit increases linearly with the growth of its radius. Therefore, the density of objects decreases with increasing distance $r$ from the observer. To overcome this issue, we used 


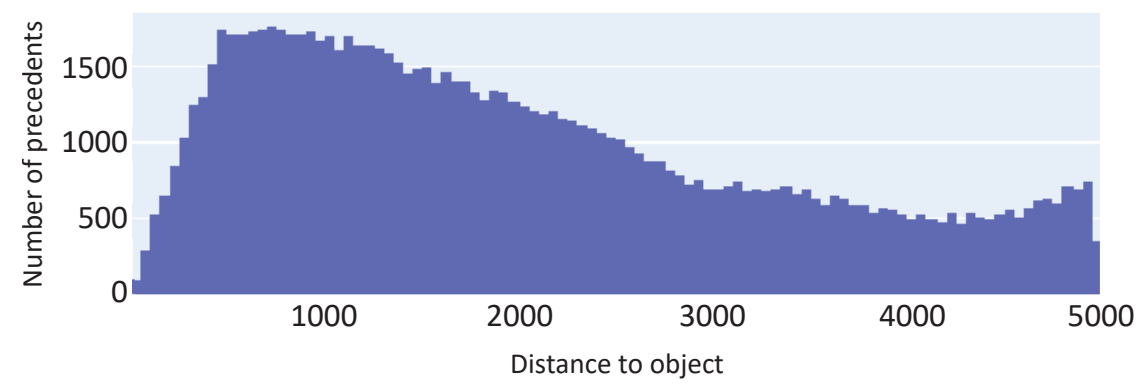

Figure 12. Precedent distribution obtained after data set generation in one pass

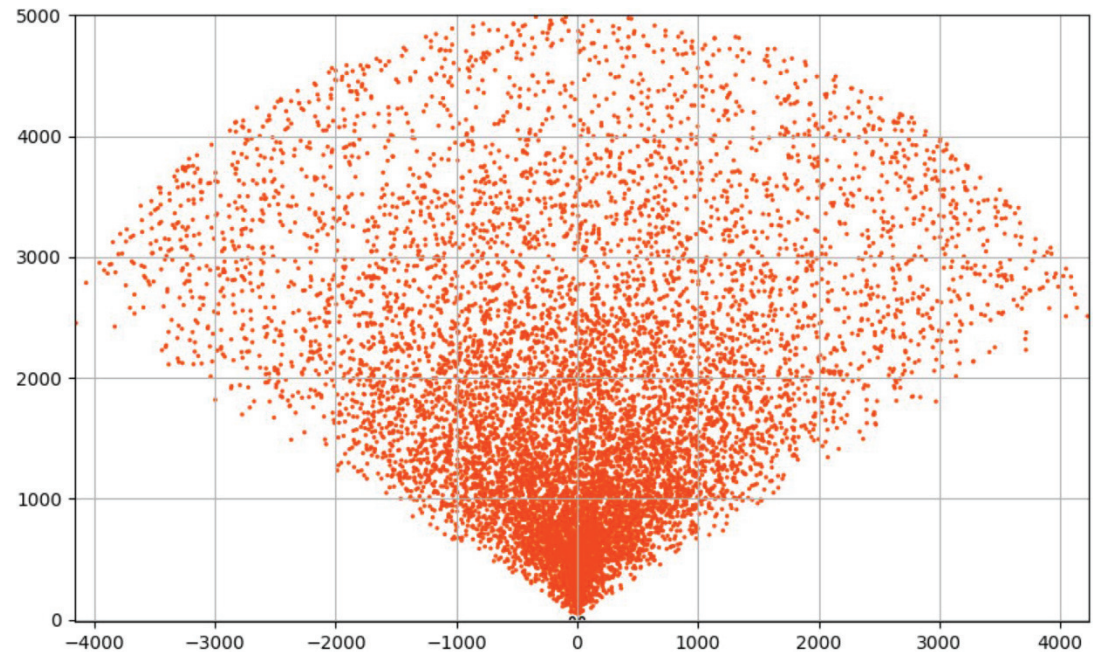

Figure 13. Spatial distribution of object centers after one pass data set generation

the method of multi-pass generation of the training data set. To do this, we divided the interval of values $[0,5000]$, specifying the distance to object, into 1000 segments of length 5 . In each $i$ th segment $(i=1, \ldots, 1000)$, using Algorithm 1 , we generated $1000+200(i-1)$ precedents. In total, we received 100900 precedents. The resulting distribution relative to the distance $r$ to the object is shown in Figure 14; the spatial distribution of the observed object centers is shown in Figure 15. The data set generated in this way is freely available at https://github.com/artem-starkov/covidsgen/dataset.

For the sake of simplicity, we decided to design two separate feedforward DNNs: the first to determine the distance and the second to determine the azimuth to a circular feature. To search for an optimal set of neural network hyperparameters, we constructed a common hypermodel for both networks (see Figure 16). The hypermodel includes the input layer, three hidden layers, and the output layer. All these layers are fully connected (have dense connections). For the input layer and all hidden layers, the activation functions to choose from are [ReLU, Tanh, Sigmoid]. The input layer has 720 neurons receiving external data: 360-bitmap from the left compound eye and 360-bitmap from the right compound eye. The output layer has a single neuron producing the ultimate result: the distance for the first DNN and the azimuth for the second DNN. For the first hidden layer, the numbers of neurons to choose from is $[2048,3072,4096,5040,5760]$. For the second and the third hidden layers, the numbers of neurons to choose from is [3072, 4096, 5120, 5760, 6480, 7200] and [2160, 2880, 3600, 4096, 5120, 6144], respectively. Based on this hypermodel, we performed a limited random search for optimal sets of neural network hyperparameters using W\&B [50]. As an optimization algorithm, we tested SGD (stochastic gradient descent) and RMSProp [51]. The batch size ranged from 4 to 64 . As a loss function, we used $M A E$ (mean absolute error) [52] calculated by the following equation:

$$
M A E=\frac{1}{n} \sum_{i=1}^{n}\left|y_{i}-x_{i}\right|,
$$




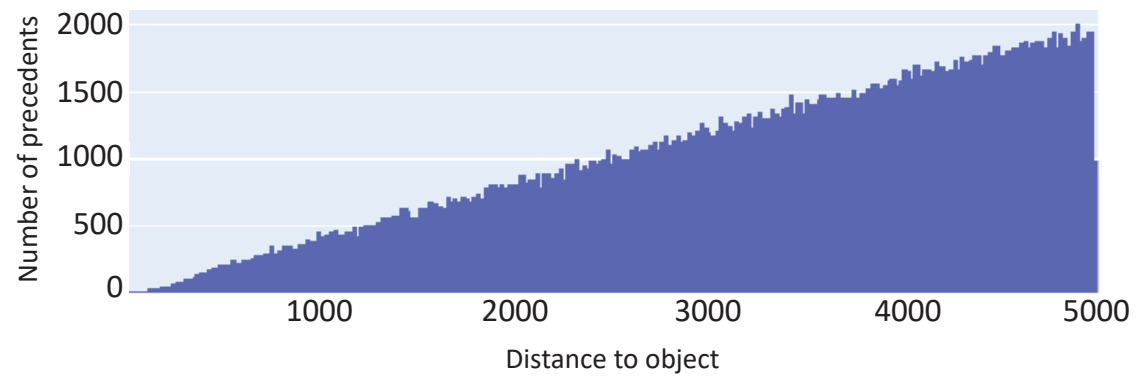

Figure 14. Precedent distribution obtained after data set generation in multiple passes (distance step = 5)

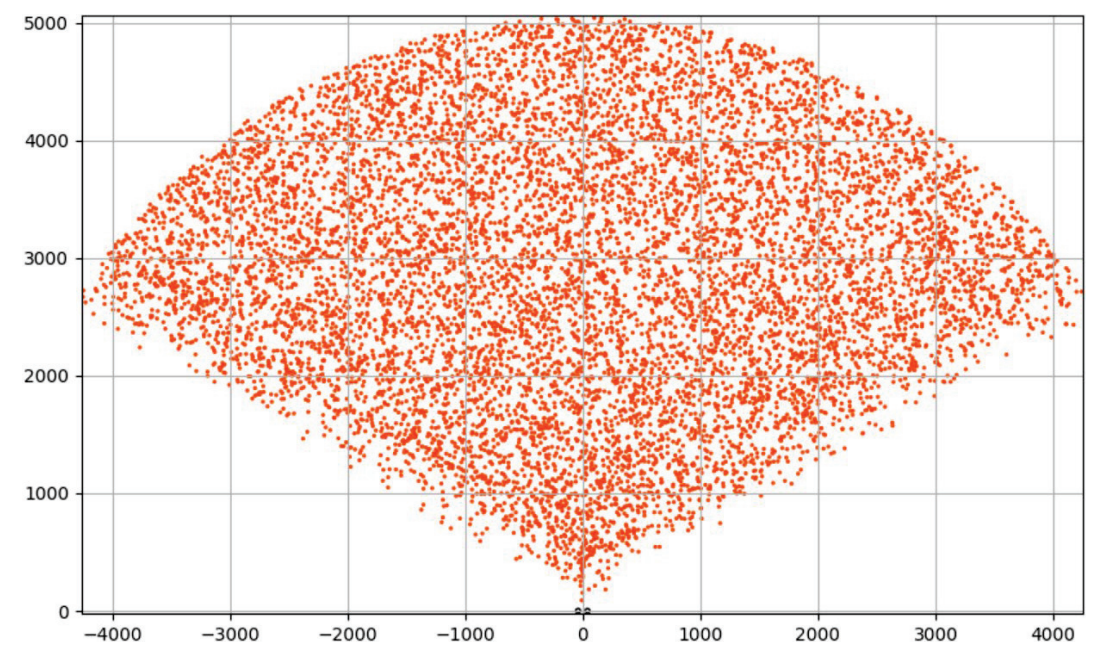

Figure 15. Spatial distribution of object centers after multiple pass data set generation

where $n$ is the number of elements of the training data set, $y_{i}$ is the DNN output, and $x_{i}$ is the true value. The generated data set of 100000 items was divided as follows:

- $\quad$ training sample: 68000 items;

- $\quad$ validation sample: 12000 items;

- $\quad$ test sample: 20000 items.

To train and test neural network models, we used the Google Colab cloud platform [53] equipped with $n$ Vidia Tesla $P 4$ graphics card. As a result, we obtained two DNNs shown in Figure 17. The final training parameters are presented in Table 3. To assess the quality of the neural network models obtained, we used the following two metrics: MAPE (mean absolute percentage error) [54] defined by the equation

$$
M A P E=\frac{100}{n} \sum_{i=1}^{n}\left|\frac{y_{i}-x_{i}}{x_{i}}\right| \text {, }
$$

and the coefficient of determination $R^{2}$ [55] defined by the equation

$$
R^{2}=1-\sum_{i=1}^{n}\left(y_{i}-x_{i}\right)^{2} / \sum_{i=1}^{n}\left(y_{i}-y_{\text {mean }}\right)^{2} \text {, }
$$

where

$$
y_{\text {mean }}=\frac{1}{n} \sum_{i=1}^{n} y_{i}
$$

The MAPE is often used in practice because of its very intuitive interpretation in terms of relative error. The coefficient of determination $R^{2}$ gives some information about the goodness of fit of a neural network model to the training data set. The models with $R^{2}>0.8$ 


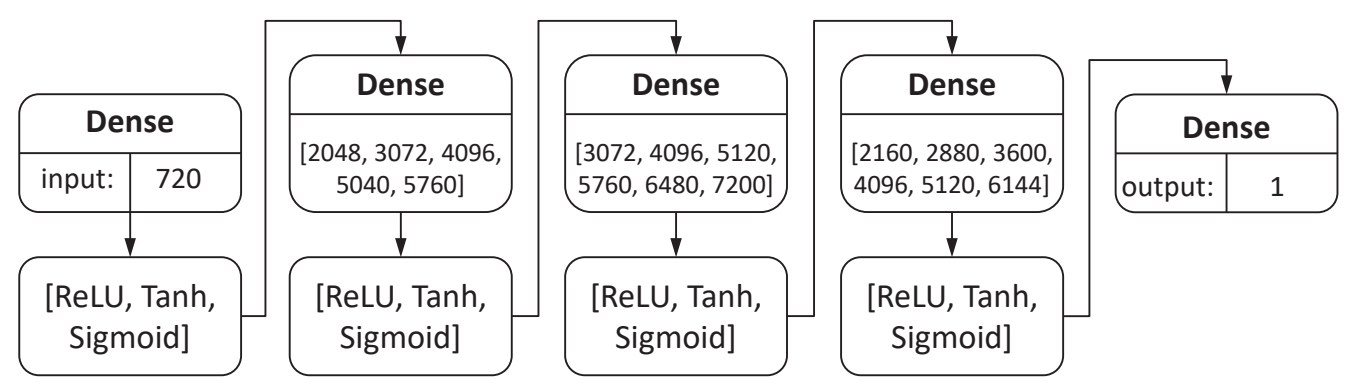

Figure 16. DNN hypermodel structure

(a) $A D$
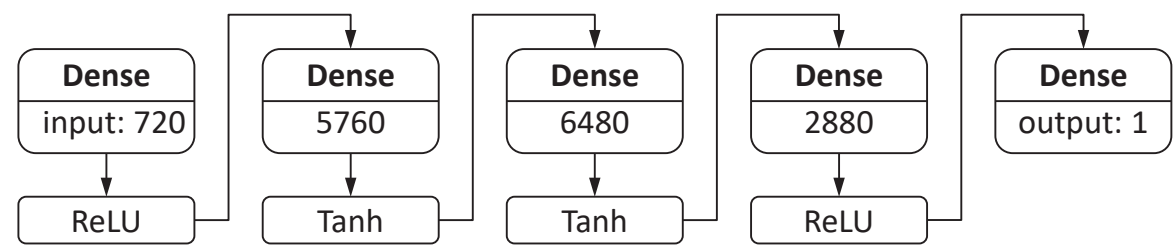

(b) DD
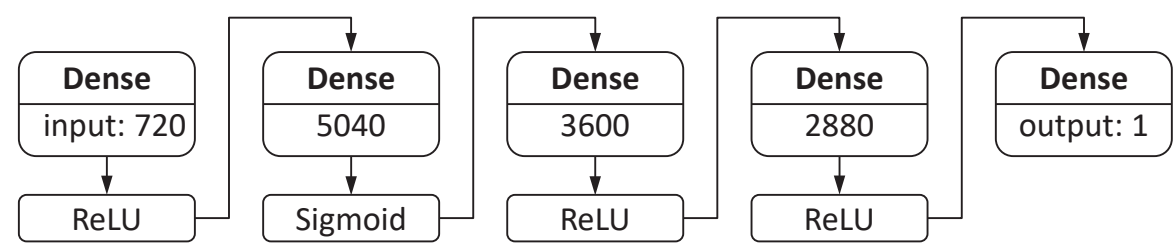

Figure 17. DNNs: (a) Azimuth detection, (b) Distance detection

can be considered quite good. The obtained values of these metrics presented in Table 3 show that both neural network models are very good.

Table 3: Final parameters of neural networks

\begin{tabular}{l|l|l}
\hline Parameter & Azimuth detection & Distance detection \\
\hline Optimizer & RMSProp & SGD \\
Batch size & 64 & 32 \\
Learning rate & 0.004 & 0.001 \\
Training time & $2 \mathrm{~h} \mathrm{3m} \mathrm{49s}$ & $48 \mathrm{~m} \mathrm{53s}$ \\
MAPE & 0.832 & 7.713 \\
$R^{2}$ & 0.998 & 0.899 \\
\hline
\end{tabular}

\section{Experiments and Discussion}

To test the accuracy of both designed neural network models, we performed a series of computational experiments. When developing the experiments, we took into account the following important point. It is well known that insects clearly see only moving objects [56]. The image motion can be a result of the movement of the object or the insect itself. The insect vision senses static images as a set of very blurred spots. Therefore, in our experiments, we simulated the movement of circular features of different radiuses along the three different trajectories shown in Figure 18.

The first type of trajectory is horizontal (see Figure 18a). Such trajectory is defined by a straight line parallel to the $\mathrm{x}$-axis and located at a distance of $r$ from the origin. The segment that is the intersection of this line with the BFV area has a length of $2 \tan \left(60^{\circ}\right) r \approx 3.5 r$. For this type of trajectory, we generated a set of circular features with a radius of $g$ in the amount of $1.5 \mathrm{r} / \mathrm{g}$, uniformly distributed along the trajectory. We fed the set of precedents constructed in this way to neural networks presented in Figure 17. The results for $g=5$, $g=50$, and $g=500$ are presented in Figure 19. Here and after Accuracy $=100 \%-$ MAPE. Diagram (a) in Figure 19 shows that the AD neural network (see Figure 17a) demonstrates great accuracy in determining an object's azimuth. For all investigated trajectories, the 


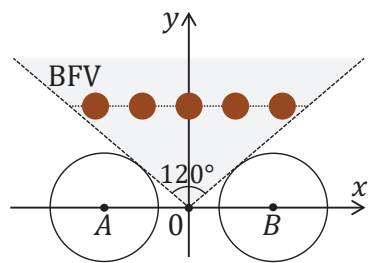

(a) Horizontal

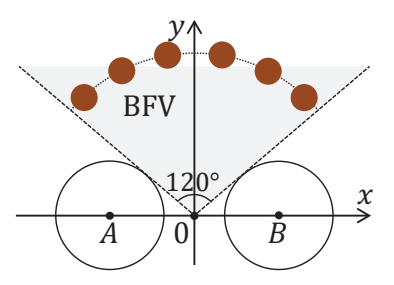

(b) Circular

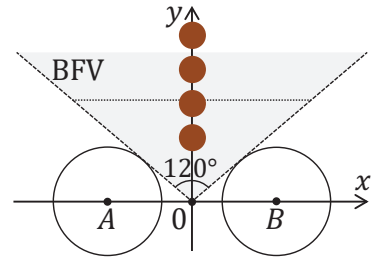

(c) Vertical

Figure 18. Types of trajectories for testing

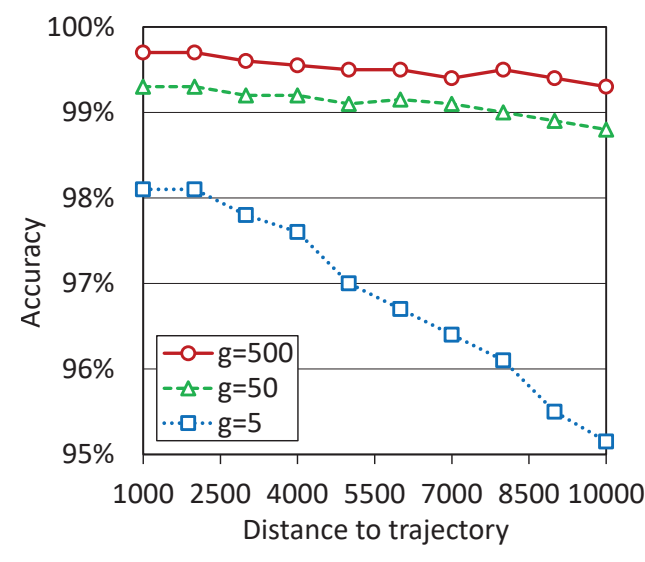

(a) Azimuth detection

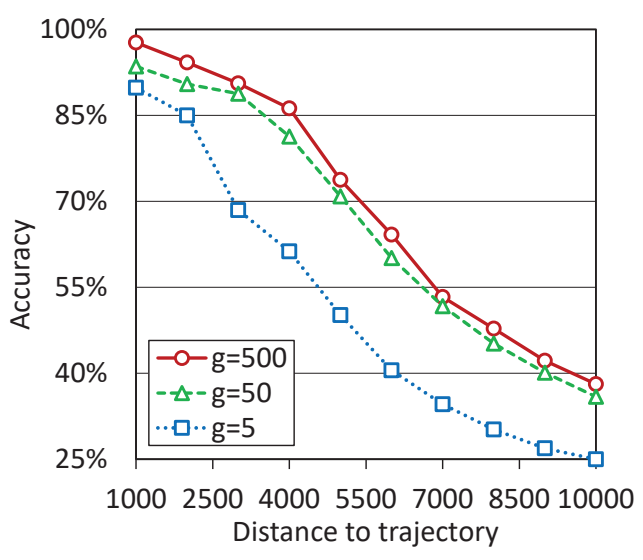

(b) Distance detection

Figure 19. Horizontal movement of object with radius $g$

accuracy of detecting the azimuth of large $(g=500)$ and medium $(g=50)$ objects is about $99 \%$; for small objects $(g=5)$, the accuracy is more than 95\%. Diagram (b) in Figure 19 shows the results obtained by the DD neural network (see Figure 17b) when detecting the distance to the object. We can see that in this case, the accuracy of determining the distance to large objects decreases from $98 \%$ to $74 \%$ with increasing distance to the trajectory from 1000 to 5000 . In this interval, the accuracy decreases from $94 \%$ to $71 \%$ for medium objects and from $90 \%$ to $50 \%$ for small objects. With increasing trajectory distance to 10000 , the accuracy of determining the distance to the object decreases to $38 \%$ for large objects, $36 \%$ for medium, and $25 \%$ for small. We should note that both neural networks learned at distances in the segment $[1000,5000]$. The experiments show that the AD neural network can work adequately at longer distances. This is explained by the fact that the object images shrink with increasing distance; small objects disappear, and large ones seem small. Therefore, the neural network can use the experience gained from training at shorter distances to detect azimuth at longer distances.

The second type of trajectory is circular (see Figure 18b). Such a trajectory is defined by a circle with radius $r$ and the center at the origin. The arc that is the intersection of this circle with the BFV area has a length of $2 \pi r 120 / 360 \approx 2.1 r$. For this type of trajectory, we generated a set of circular features with a radius of $g$ in the amount of $r / g$, uniformly distributed along the trajectory. We fed the set of precedents constructed in this way to the AD neural network (see Figure 17a). The results are presented in Figure 20. We can see that the AD neural network demonstrates great accuracy in determining an object's azimuth. For all investigated trajectories, the accuracy of detecting the azimuth of large $(g=500)$ and medium $(g=50)$ objects is more then $99 \%$; for small objects $(g=5)$, the accuracy is more than $97 \%$. The results obtained in this experiment look a little better than ones obtained when the object moves along a horizontal trajectory (see Figure 19a). This is because the ends of the horizontal segment of the trajectory are farther from the observer than its middle. In contrast, in the case of circular trajectory, the distance to the observer is always constant.

The third type of trajectory is vertical (see Figure 18c). Vertical trajectory coincides with the central axis of the BFV area. In our experiment, this trajectory has a length of 


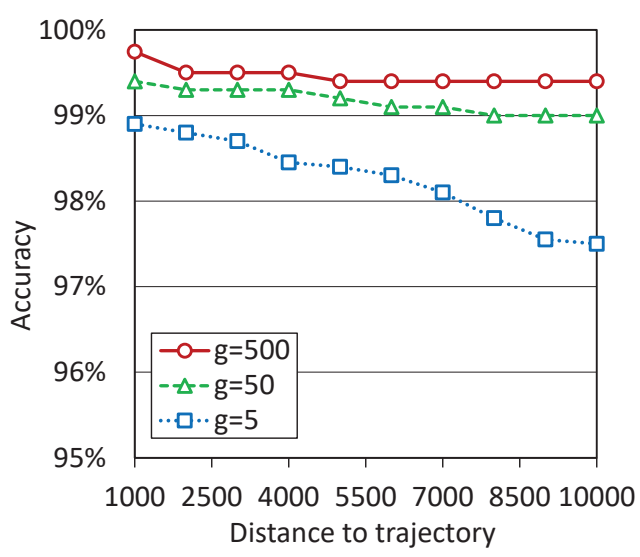

Figure 20. Azimuth to object moving along circular trajectory

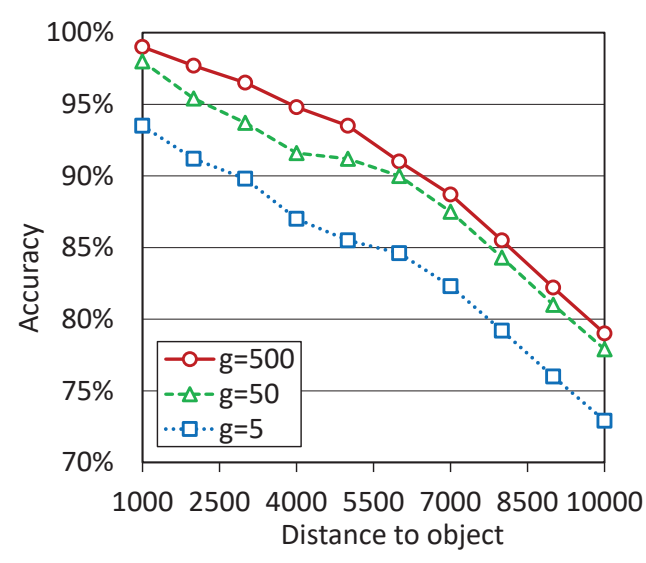

Figure 21. Distance to object moving along vertical trajectory

9000. For this type of trajectory, we generated a set of circular features with a radius of $g$ in the amount of $4500 / g$, uniformly distributed along the trajectory. We fed the set of precedents constructed in this way to the DD neural network (see Figure 17b). The results are presented in Figure 21. The diagram shows that the accuracy of distance detection for all objects does not decrease below $70 \%$ in segment [1000, 10000]. Thus, the results obtained in this experiment look much better than ones obtained when the object moves along a horizontal trajectory (see Figure 19b). This is because the objects, in the case of vertical trajectory, are located on the central axis of the BFV area. We can conclude that to detect the distance to the object more accurately, the binocular compound eye vision system must turn frontally to the observed object.

At the end of this section, we will discuss the following issues.

1. What is the main contribution of this article?

2. What are the advantages and disadvantages of the proposed approach compared to other known methods?

3. Is the 2D model of compound eye vision applicable in practice?

4. What are the future applications of the proposed model?

5. What confirms the adequacy of the model?

Let us start answering the indicated questions. The main contribution of the article is a mathematical model of planar compound eye vision and a method for detecting the azimuth and distance to the observed object, which does not require the use of active sensors. Another important result is that DNNs based on the proposed model are able to detect azimuth and distance with high accuracy.

The advantage of the proposed method is a potentially more accurate measurement of azimuth and distance to the object compared to other known methods using passive sensors. The disadvantage of the described method is its inapplicability for the case when the observer and the object are static relative to each other. We will explain below why.

The 2D model of compound eye vision can be used to develop ground autonomous mobile robots. To operate on a surface, it is enough for the robot to have two planar compound eyes, each of which has one row of optical sensors (artificial ommatidia) installed in a circle. Similar implementations are known in the literature (see, for example, [14]). The optical sensor of the artificial compound eye transmits a signal to the neural network only when it detects an increase in light intensity. The signal will be especially strong if the adjacent optical sensor simultaneously detects a decrease in light intensity. In such a way, the neural network can sharply see the bounds of a moving object. That's why the object must move relative to the compound eye vision system in order to be detected.

The future applications of the proposed model are the following. First, the 2D model of compound eye vision can serve as a basis for designing physical optical systems for groundbased mobile robots. Such robots can participate in the mitigation of the consequences of 
accidents at nuclear power plants and extinguish fires at industrial facilities. Second, the proposed model makes it possible to construct an synthetic data set for prior supervised learning a neural network to detect the azimuth and distance to the observed object. After that, the pre-trained neural network is embedded in the physical prototype, and the final reinforcement learning is performed.

To confirm the adequacy of the developed model, we must implement it in the form of a physical prototype and check its operability. This is the subject of our future research. Nevertheless, when developing this model, we used the bionic principles inspired by the compound eyes of insects. To simulate a binocular compound eye system, we used the parameters comparable to the proportions of the robber fly vision system. This allows us to hope that the adequacy of the model will be confirmed in practice.

\section{Conclusion}

In this paper, we proposed a 2D model of the binocular compound eye vision inspired by insects eyes. The model includes two circular compound eyes located on a plane. The compound eye includes one row of ommatidia (optical sensors), arranged in a circle. The ommatidia are represented by equal-sized isosceles triangles. In the model, the ommatidium field of view is defined as a solid angle bounded by its legs. A ray tracing model was built on this basis. Using this model, an algorithm was developed for generating labeled data sets for training artificial neural networks capable of determining the distance and azimuth to the observed objects. Using the data sets generated by this algorithm, we designed and trained two feedforward deep neural networks: one to determine the distance and the other to determine the azimuth to circular features. To test the accuracy of both designed neural network models, we simulated the movement of circular features along different trajectories. Computational experiments have shown that the designed networks can detect azimuth with an accuracy of about $99 \%$ and the distance with an accuracy of about $80 \%$ for medium and large circular features.

Our future research directions on this subject are the following. We plan to investigate the dependence of the accuracy of azimuth and distance detection on the following parameters of the binocular compound eye vision system: the diameter of compound eyes, the distance between the eyes, the number of ommatidia in the eye. We also plan to create a prototype of a planar binocular compound eye vision system to validate the described mathematical model. The same prototype will be used for reinforcement learning the neural networks trained on synthetic data in the present study. Our next goal is to create and investigate a three-dimensional model of a binocular compound eye vision system focused on azimuth and distance detection.

Author Contributions: All authors contributed equally to the main text. All authors have read and agreed to the published version of the manuscript.

Funding: This research was supported by the Ministry of Science and Higher Education of the Russian Federation (gov. order FENU-2020-0022).

Conflicts of Interest: The authors declare no conflict of interest.

\section{References}

1. Ben-Ari, M.; Mondada, F. Robots and Their Applications. In Elements of Robotics; Springer: Cham, 2018; chapter 1, pp. 1-20. doi:10.1007/978-3-319-62533-1_1.

2. Jahn, U.; Hess, D.; Stampa, M.; Sutorma, A.; Rohrig, C.; Schulz, P.; Wolff, C. A Taxonomy for Mobile Robots: Types, Applications, Capabilities, Implementations, Requirements, and Challenges. Robotics 2020, 9, Article 109. doi:10.3390/ROBOTICS9040109.

3. Ben-Ari, M.; Mondada, F. Sensors. In Elements of Robotics; Springer: Cham, 2018; chapter 2, pp. 21-37. doi:10.1007/978-3-31962533-1_2.

4. Mahajan, A.; Walworth, M. 3-D position sensing using the differences in the time-of-flights from a wave source to various receivers. IEEE Transactions on Robotics and Automation 2001, 17, 91-94. doi:10.1109/70.917087.

5. Siegwart, R.; Nourbakhsh, I.R.; Scaramuzza, D. Introduction to Autonomous Mobile Robots, 2 ed.; MIT Press: Cambridge, Massachusetts; London, England, 2011; p. 488. 
6. Rodriguez-Quinonez, J.C.; Sergiyenko, O.; Flores-Fuentes, W.; Rivas-lopez, M.; Hernandez-Balbuena, D.; Rascon, R.; Mercorelli, P. Improve a $3 \mathrm{D}$ distance measurement accuracy in stereo vision systems using optimization methods' approach. Opto-electronics Review 2017, 25, 24-32. doi:10.1016/j.opelre.2017.03.001.

7. Jeong, C.J.; Park, G.M. Real-time Auto Tracking System using PTZ Camera with DSP. International journal of advanced smart convergence 2013, 2, 32-35. doi:10.7236/IJASC.2013.2.1.032.

8. Davis, J.D.; Barrett, S.F.; Wright, C.H.; Wilcox, M. A bio-inspired apposition compound eye machine vision sensor system. Bioinspiration and Biomimetics 2009, 4, Article 046002. doi:10.1088/1748-3182/4/4/046002.

9. Wu, S.; Jiang, T.; Zhang, G.; Schoenemann, B.; Neri, F.; Zhu, M.; Bu, C.; Han, J.; Kuhnert, K.D. Artificial compound eye: a survey of the state-of-the-art. Artificial Intelligence Review 2017, 48, 573-603. doi:10.1007/s10462-016-9513-7.

10. Land, M.F. Visual acuity in insects. Annual Review of Entomology 1997, 42, 147-177. doi:10.1146/ANNUREV.ENTO.42.1.147.

11. Nakayama, K. Biological image motion processing: A review. Vision Research 1985, 25, 625-660. doi:10.1016/0042-6989(85)90171-3.

12. Phan, H.L.; Yi, J.; Bae, J.; Ko, H.; Lee, S.; Cho, D.; Seo, J.M.; Koo, K.i. Artificial Compound Eye Systems and Their Application: A Review. Micromachines 2021, 12, Article 847. doi:10.3390/MI12070847.

13. Franceschini, N.; Pichon, J.; Blanes, C. From insect vision to robot vision. Philosophical Transactions of the Royal Society of London. Series B: Biological Sciences 1992, 337, 283-294. doi:10.1098/rstb.1992.0106.

14. Franceschini, N. From Fly Vision to Robot Vision: Re-Construction as a Mode of Discovery. In Sensors and Sensing in Biology and Engineering; Barth, F.G.; Humphrey, J.A.; Secomb, T.W., Eds.; Springer: Vienna, 2003; chapter 16, pp. 223-236. doi:10.1007/978-37091-6025-1_16.

15. Floreano, D.; Pericet-Camara, R.; Viollet, S.; Ruffier, F.; Bruckner, A.; Leitel, R.; Buss, W.; Menouni, M.; Expert, F.; Juston, R.; Dobrzynski, M.K.; L'Eplattenier, G.; Recktenwald, F.; Mallot, H.A.; Franceschini, N. Miniature curved artificial compound eyes. Proceedings of the National Academy of Sciences of the United States of America 2013, 110, 9267-9272. doi:10.1073/pnas.1219068110.

16. Zhu, L.; Zhang, Y.L.; Sun, H.B.; Zhu, L.; Zhang, Y.L.; Sun, H.B. Miniaturising artificial compound eyes based on advanced micronanofabrication techniques. Light: Advanced Manufacturing 2021, 2, 84-100. doi:10.37188/LAM.2021.007.

17. Prieto, A.; Prieto, B.; Ortigosa, E.M.; Ros, E.; Pelayo, F.; Ortega, J.; Rojas, I. Neural networks: An overview of early research, current frameworks and new challenges. Neurocomputing 2016, 214, 242-268. doi:10.1016/j.neucom.2016.06.014.

18. Schmidhuber, J. Deep learning in neural networks: An overview. Neural Networks 2015, 61, 85-117. doi:10.1016/j.neunet.2014.09.003.

19. Zou, A.M.; Hou, Z.G.; Fu, S.Y.; Tan, M. Neural Networks for Mobile Robot Navigation: A Survey. Advances in Neural Networks - ISNN 2006. Third International Symposium on Neural Networks, Chengdu, China, May 28 - June 1, 2006, Proceedings, Part II. Lecture Notes in Computer Science, vol. 3972; Wang, J.; Yi, Z.; Zurada, J.M.; Lu, B.L.; Yin, H., Eds.; Springer: Berlin, Heidelberg, New York, 2006; pp. 1218-1226. doi:10.1007/11760023_177.

20. Yu, J.; Su, Y.; Liao, Y. The Path Planning of Mobile Robot by Neural Networks and Hierarchical Reinforcement Learning. Frontiers in Neurorobotics 2020, 14, Article 63. doi:10.3389/FNBOT.2020.00063.

21. Medvedev, M.; Kadhim, A.; Brosalin, D. Development of the Neural-Based Navigation System for a Ground-Based Mobile Robot. 2021 The 7th International Conference on Mechatronics and Robotics Engineering (ICMRE 2021). IEEE, 2021, pp. 35-40. doi:10.1109/ICMRE51691.2021.9384825.

22. Sharma, V.; Mir, R.N. A comprehensive and systematic look up into deep learning based object detection techniques: A review. Computer Science Review 2020, 38, Article 100301. doi:10.1016/j.cosrev.2020.100301.

23. de Menezes, R.S.T.; Magalhaes, R.M.; Maia, H. Object Recognition Using Convolutional Neural Networks. In Recent Trends in Artificial Neural Networks - from Training to Prediction; Sadollah, A.; Travieso-Gonzalez, C.M., Eds.; IntechOpen, 2020 ; chapter 5. doi:10.5772/INTECHOPEN.89726.

24. Chi, K.H.; Lee, M.F.R. Obstacle avoidance in mobile robot using neural network. 2011 International Conference on Consumer Electronics, Communications and Networks, CECNet 2011 - Proceedings, 2011, pp. 5082-5085. doi:10.1109/CECNET.2011.5768815.

25. Feng, S.; Sebastian, B.; Ben-Tzvi, P. A Collision Avoidance Method Based on Deep Reinforcement Learning. Robotics 2021, 10, Article 73. doi:10.3390/ROBOTICS10020073.

26. Kocic, J.; Jovicic, N.; Drndarevic, V. An End-to-End Deep Neural Network for Autonomous Driving Designed for Embedded Automotive Platforms. Sensors 2019, 19, Article 2064. doi:10.3390/S19092064.

27. Bojarski, M.; Yeres, P.; Choromanska, A.; Choromanski, K.; Firner, B.; Jackel, L.; Muller, U. Explaining How a Deep Neural Network Trained with End-to-End Learning Steers a Car. arXiv:1704.07911 [cs.CV]. 2017.

28. Brigato, L.; Iocchi, L. A Close Look at Deep Learning with Small Data. 2020 25th International Conference on Pattern Recognition (ICPR). IEEE, 2021, pp. 2490-2497. doi:10.1109/ICPR48806.2021.9412492.

29. Iuzzolino, M.L.; Walker, M.E.; Szafir, D. Virtual-to-Real-World Transfer Learning for Robots on Wilderness Trails. 2018 IEEE/RSJ International Conference on Intelligent Robots and Systems (IROS). IEEE, 2018, pp. 576-582. doi:10.1109/IROS.2018.8593883.

30. Liu, F.; Diao, X.; Li, L.; Hao, Y.; Jiao, Z. Fabrication and Characterization of Inhomogeneous Curved Artificial Compound Eye. Micromachines 2018, 9, Article 238. doi:10.3390/MI9050238.

31. Jungel, M.; Mellmann, H.; Spranger, M. Improving Vision-Based Distance Measurements Using Reference Objects. RoboCup 2007: Robot Soccer World Cup XI. RoboCup 2007. Lecture Notes in Computer Science, vol. 5001; Visser, U.; Ribeiro, F.; Ohashi, T.; Dellaert, F., Eds.; Springer: Berlin, Heidelberg, 2007; pp. 89-100. doi:10.1007/978-3-540-68847-1_8.

32. Shiu, Y.C.; Ahmad, S. 3D location of circular and spherical features by monocular model-based vision. Proceedings of the IEEE International Conference on Systems, Man and Cybernetics. Vol. 2. IEEE, 1989, pp. 576-581. doi:10.1109/ICSMC.1989.71362. 
33. Safaee-Rad, R.; Tchoukanov, I.; Smith, K.C.; Benhabib, B. Three-Dimensional Location Estimation of Circular Features for Machine Vision. IEEE Transactions on Robotics and Automation 1992, 8, 624-640. doi:10.1109/70.163786.

34. Li, L. Building an accurate 3D model of a circular feature for robot vision. Opto-electronics Review 2012, 20. doi:10.2478/s11772012-0017-y.

35. Sun, X.; Jiang, Y.; Ji, Y.; Fu, W.; Yan, S.; Chen, Q.; Yu, B.; Gan, X. Distance Measurement System Based on Binocular Stereo Vision. IOP Conference Series: Earth and Environmental Science 2019, 252, Article 052051. doi:10.1088/1755-1315/252/5/052051.

36. Mustafah, Y.M.; Azman, A.W.; Ani, M.H. Object distance and size measurement using stereo vision system. Advanced Materials Research 2013, 622-623, 1373-1377. doi:10.4028/WWW.SCIENTIFIC.NET/AMR.622-623.1373.

37. Sanchez-Ferreira, C.; Mori, J.Y.; Farias, M.C.Q.; Llanos, C.H. A real-time stereo vision system for distance measurement and underwater image restoration. Journal of the Brazilian Society of Mechanical Sciences and Engineering 2016, 38, $2039-2049$. doi:10.1007/S40430-016-0596-5.

38. Dandil, E.; Cevik, K.K. Computer Vision Based Distance Measurement System using Stereo Camera View. 3rd International Symposium on Multidisciplinary Studies and Innovative Technologies, ISMSIT 2019 - Proceedings. IEEE, 2019, pp. 1-4. doi:10.1109/ISMSIT.2019.8932817.

39. Zaarane, A.; Slimani, I.; Al Okaishi, W.; Atouf, I.; Hamdoun, A. Distance measurement system for autonomous vehicles using stereo camera. Array 2020, 5, 100016. doi:10.1016/j.array.2020.100016.

40. Zhang, Z. Determining the Epipolar Geometry and its Uncertainty: A Review. International Journal of Computer Vision 1998, 27,161-195. doi:10.1023/A:1007941100561.

41. Szeliski, R. Computer Vision: Algorithms and Applications; Texts in Computer Science, Springer: London, Dordrecht, Heidelberg, New York, 2011; p. 812. doi:10.1007/978-1-84882-935-0.

42. Poggi, M.; Kim, S.; Tosi, F.; Kim, S.; Aleotti, F.; Min, D.; Sohn, K.; Mattoccia, S. On the confidence of stereo matching in a deep-learning era: a quantitative evaluation. Preprint. IEEE Transactions on Pattern Analysis and Machine Intelligence 2021. doi:10.1109/TPAMI.2021.3069706.

43. Hanning, T. High Precision Camera Calibration; Vieweg+Teubner, 2011; p. 212. doi:10.1007/978-3-8348-9830-2.

44. Chen, Y.; Jin, X.; Dai, Q. Distance measurement based on light field geometry and ray tracing. Optics Express 2017, 25, 76. doi:10.1364/oe.25.000059.

45. Lumsdaine, A.; Georgiev, T. The focused plenoptic camera. 2009 IEEE International Conference on Computational Photography (ICCP). IEEE, 2009, pp. 1-8. doi:10.1109/ICCPHOT.2009.5559008.

46. Heinze, C.; Spyropoulos, S.; Hussmann, S.; Perwass, C. Automated Robust Metric Calibration Algorithm for Multifocus Plenoptic Cameras. IEEE Transactions on Instrumentation and Measurement 2016, 65, 1197-1205. doi:10.1109/TIM.2015.2507412.

47. Sardemann, H.; Maas, H.G. On the accuracy potential of focused plenoptic camera range determination in long distance operation. ISPRS Journal of Photogrammetry and Remote Sensing 2016, 114, 1-9. doi:10.1016/J.ISPRSJPRS.2016.01.012.

48. Ashley, D. Foundation Dynamic Web Pages with Python; Apress: Austin, TX, USA, 2020; p. 213. doi:10.1007/978-1-4842-6339-6.

49. Wardill, T.J.; Fabian, S.T.; Pettigrew, A.C.; Stavenga, D.G.; Nordstr?m, K.; Gonzalez-Bellido, P.T. A Novel Interception Strategy in a Miniature Robber Fly with Extreme Visual Acuity. Current Biology 2017, 27, 854-859. doi:10.1016/j.cub.2017.01.050.

50. Biewald, L. Experiment Tracking with Weights and Biases. https://docs.wandb.ai/ 2020.

51. Goodfellow, I.; Bengio, Y.; Courville, A. Deep Learning (Adaptive Computation and Machine Learning); MIT Press, 2016 ; p. 800.

52. Willmott, C.J.; Matsuura, K. Advantages of the mean absolute error (MAE) over the root mean square error (RMSE) in assessing average model performance. Climate Research 2005, 30, 79-82. doi:10.3354/CR030079.

53. Bisong, E. Building Machine Learning and Deep Learning Models on Google Cloud Platform; Apress: Berkeley, CA, 2019; pp. XXIX, 709. doi:10.1007/978-1-4842-4470-8.

54. de Myttenaere, A.; Golden, B.; Le Grand, B.; Rossi, F. Mean Absolute Percentage Error for regression models. Neurocomputing 2016, 192, 38-48. doi:10.1016/J.NEUCOM.2015.12.114.

55. Barten, A.P. The coefficient of determination for regression without a constant term. In The Practice of Econometrics. International Studies in Economics and Econometrics, vol. 15; Heijmans, R.; Neudecker, H., Eds.; Springer: Dordrecht, 1987; pp. 181-189. doi:10.1007/978-94-009-3591-4_12.

56. Borst, A.; Haag, J.; Reiff, D.F. Fly Motion Vision. Annual Review of Neuroscience 2010, 33, 49-70. doi:10.1146/ANNUREV-NEURO060909-153155. 